\title{
Nitric oxide production is involved in maintaining energy state in Alfalfa (Medicago sativa L.) nodulated roots under both salinity and flooding
}

\author{
Fatma Aridhi $^{1,2} \cdot$ Hajer Sghaier $^{1} \cdot$ Allyzée Gaitanaros $^{2} \cdot$ Ayda Khadri $^{1} \cdot$ Samira Aschi-Smiti $^{1} \cdot$ Renaud Brouquisse $^{2}$ BD
}

Accepted: 8 July 2020 / Published online: 16 July 2020

\begin{abstract}
Main conclusion In Medicago sativa nodulated roots, NR-dependent NO production is involved in maintaining energy state, presumably through phytoglobin NO respiration, under both salinity and hypoxia stress.
\end{abstract}

\begin{abstract}
The response to low and average salinity stress and to a 5 day-long flooding period was analyzed in $M$. sativa nodulated roots. The two treatments result in a decrease in the biological nitrogen fixation capacity and the energy state (evaluated by the ATP/ADP ratio), and conversely in an increase nitric oxide (NO) production. Under salinity and hypoxia treatments, the use of either sodium tungstate, an inhibitor of nitrate reductase (NR), or carboxy-PTIO, a NO scavenger, results in a decrease in NO production and ATP/ADP ratio, meaning that NR-dependent NO production participates to the maintenance of the nodulated roots energy state.
\end{abstract}

Keywords Hypoxia · Legume · Nitrogen-fixing symbiosis · Phytoglobin NO respiration · Salt stress

Renaud Brouquisse

renaud.brouquisse@inrae.fr

Fatma Aridhi

aridhifatma@gmail.com

Hajer Sghaier

sghaier.hajer@hotmail.com

Allyzée Gaitanaros

allyzee.gaitanaros@hotmail.fr

Ayda Khadri

khadriayda@yahoo.fr

Samira Aschi-Smiti

samira.smiti@planet.tn

1 Unité de Recherche d'Ecologie Végétale, Faculté des Sciences de Tunis, Université de Tunis El Manar, Campus Universitaire Farhat Hached, Tunis, Tunisia

2 UMR INRAE 1355, CNRS 7254, Université Côte d'Azur, Institut Sophia Agrobiotech, Sophia Antipolis, France

\author{
Abbreviations \\ ARA Acetylene reducing activity \\ BNF Biological nitrogen fixation \\ cPTIO 2-[4-carboxyphenyl]-4,4,5,5-tetramethylimida- \\ zoline-1-oxyl-3-oxide \\ DAF-2 4,5-diaminofluorescein \\ NR Nitrate reductase \\ Phytogb Phytoglobin
}

\section{Introduction}

Nitric oxide (NO) is a reactive gaseous molecule with a broad spectrum of regulatory functions in plant growth and development (Besson-Bard et al. 2008), and response to biotic (Hichri et al. 2015; Thalineau et al. 2016) and abiotic stresses, including salinity and hypoxia (Blokhina and Fagerstedt 2010; Simontacchi et al. 2015). Under salt stress, enhancement of $\mathrm{NO}$ production is accompanied by the exclusion of $\mathrm{Na}^{+}$and retention of $\mathrm{K}^{+}$, through increased membrane $\mathrm{H}^{+}$-ATPase and $\mathrm{H}^{+}$-PPase activities (Zhang et al. 2006; Wang et al. 2009; Zhao et al. 2018). NO induces the expression of genes related to osmotic adjustment, heatshock protein, and ROS-scavenging processes (Uchida et al. 2002; Kopyra and Gwózdz 2003). It contributes to the 
maintenance of cellular redox homeostasis by controlling the NADPH level (Liu et al. 2007) and by inducing antioxidant enzyme activities (Tanou et al. 2009). Overall speaking, NO protects plants by helping them to control water status, maintain ionic homeostasis and reduce oxidative damage imposed during salt stress (Molassiotis et al. 2010; Simontacchi et al. 2015).

On the other hand, increased NO production is a hallmark of plant response to flooding and hypoxia (Blokhina and Fagerstedt 2010; Gupta and Igamberdiev 2011). Hypoxia compromises mitochondrial respiration and leads to an insufficiency in ATP for energy-demanding processes (Bailey-Serres and Voesenek 2008). In these conditions, NO contributes to the recycling of NADH and the synthesis of ATP through the setting up of an alternative respiration called "phytoglobin (Phytogb)-NO respiration" (Stoimenova et al. 2007; Igamberdiev and Hill 2009; Gupta and Igamberdiev 2011). NO is also essential for the development of lysigenous aerenchyma that enhance $\mathrm{O}_{2}$ diffusion along with the roots (Wany et al. 2017). Furthermore, the signaling role of $\mathrm{NO}$ in the perception and the response to hypoxia was recently elucidated in Arabidopsis thaliana (Hartman et al. 2019). An increase in NO production, therefore, appears to be a common response to both salt stress and hypoxia.

Exposure of nitrogen-fixing legumes to high salt concentrations resulted in a rapid decrease in nitrogenase activity and nodule respiration, and this decrease was compensated by raising $\mathrm{pO}_{2}$ (Serraj et al. 1994; Serraj and Drevon 1998). Similarly, by comparing nodule respiration of various legumes under $\mathrm{NaCl}$ treatments, Delgado et al. (1994) concluded that under low salinity the supply of $\mathrm{O}_{2}$ to nodules is the limiting factor of the biological nitrogen fixation (BNF). These observations suggest that under salt stress, the nodulated roots of legumes could also face hypoxia and that this could limit BNF.

To test this hypothesis, using the Medicago sativa legume model, we analyzed BNF, NO production and energy state in the nodulated roots of plants grown in the presence of low to average salinity and subjected to short-term flooding. Our data show that, under moderate salt stress as under flooding, a nitrate reductase (NR)-dependent NO production is induced which participates to the maintenance of the energy state of the root system.

\section{Materials and methods}

\section{Biological material and growth conditions}

Seeds of Medicago sativa (var. Siriver; obtained from Espave Vert Tunesie, Tunis, Tunesia) were sterilized and germinated as previously described (del Giudice et al. 2011). Five-day-old germinated seeds were transplanted in pots ( 2 plants/pot) filled with $250 \mathrm{~cm}^{3}$ of B5 sand (0.6-1.6 $\mathrm{mm}$ diameter). Plants growth conditions and nutrient solution are described in Sghaier et al. (2020). To set salinity conditions, nutrient solution also contained either $0.2 \mathrm{mM} \mathrm{KNO}_{3}$ (Control, Ctrl), $0.2 \mathrm{mM} \mathrm{KNO}_{3}$ and $20 \mathrm{mM} \mathrm{NaCl}$ (Treatment 20, T20), or $0.9 \mathrm{mM} \mathrm{KNO}_{3}$ and $50 \mathrm{mM} \mathrm{NaCl}$ (Treatment 50, T50). Plants were inoculated 7 days after transplanting with Ensifer meliloti 2011 bacteria (Sghaier et al. 2020).

In the first set of experiments (experiment 1), at 28 days post-inoculation (dpi), half of the Ctrl, T20 and T50 plants were subjected to flooding for 5 days by submerging pots in their respective nutrient solutions to sand level (Fig. S1a). Non-flooded plants were watered with nutrient solutions at 28 and 31 dpi (Fig. S1b). At 32 dpi, nodulated roots were harvested and either used for growth parameters analysis ( 9 plants/assay), $\mathrm{N}_{2}$-fixing capacity (2 plants/assay), NO production (2 plants/assay), or immediately frozen in liquid $\mathrm{N}_{2}$, ground in powder and stored at $-80{ }^{\circ} \mathrm{C}$ until analysis of protein and adenine nucleotide contents ( 2 plants/assay).

In the second set of experiments (experiment 2), at $28 \mathrm{dpi}$, the plants were transferred either into syringes filled with $35 \mathrm{ml}$ nutrient solution and traversed by a humidified airflow (normoxia, Fig. S2a and b) or into glass tubes filled with $40 \mathrm{ml}$ nutrient solution previously flushed with a mixture of 4.5-95.5\%: $\mathrm{O}_{2}-\mathrm{N}_{2}$ (hypoxia, Fig. S2c and d). The plants (2 plants/syringe or tube) were cultured either in normoxia or hypoxia for 5 days. To compensate for the evaporation, syringes and tubes were supplemented daily with nutrient solution. One day before harvest, nutrient solutions were supplemented with either $0.1 \mathrm{mM} 2$-[4-carboxyphenyl]4,4,5,5-tetramethylimidazoline-1-oxyl-3-oxide (cPTIO), a scavenger of $\mathrm{NO}$, or $1 \mathrm{mM}$ sodium tungstate (Tg), an inhibitor of NR, or water (control). At $32 \mathrm{dpi}$, nodulated root was promptly excised, and used for the analysis of NO production $(3 \times 2$ plants/assay), or immediately frozen in liquid $\mathrm{N}_{2}$, ground in powder and stored at $-80{ }^{\circ} \mathrm{C}$ until adenine nucleotide measurements $(3 \times 2$ plants/assay).

\section{Vegetative growth analysis}

Root systems were washed in distilled water, blotted on filter paper and weighted. Dry weights were measured after drying of samples for $48 \mathrm{~h}$ at $70{ }^{\circ} \mathrm{C}$.

\section{Biological nitrogen-fixing capacity}

BNF capacity of nodulated roots was determined in vivo by measuring the acetylene reducing activity (ARA) as previously described (Pierre et al. 2014). 


\section{Measurement of NO production}

Segments $(2 \mathrm{~cm}$-long) of nodulated roots were incubated in the dark, at $23{ }^{\circ} \mathrm{C}$, in $5 \mathrm{~mL}$ tubes containing $2 \mathrm{~mL}$ of detection medium (10 mM Tris- $\mathrm{HCl} \mathrm{pH} 7.5,10 \mathrm{mM} \mathrm{KCl})$ in the presence of $10 \mu \mathrm{M} 4,5$-diaminofluorescein (DAF-2, Coger, with excitation at $495 \mathrm{~nm}$ and emission at $515 \mathrm{~nm}$ ) fluorescent probe. NO production and controls were carried out as in Horchani et al. (2011). In addition, to test the specificity of the DAF-2 probe, NO production was also analysed with the $\mathrm{Cu}$ (II) fluorescein (CuFL) fluorescent probe (Strem Chemicals, with excitation at $495 \mathrm{~nm}$ and emission at $515 \mathrm{~nm}$ ), which is known to react rapidly and specifically with NO itself (Lim et al. 2006).

\section{Extraction and measurement of adenine nucleotides and proteins}

Adenine nucleotides were extracted essentially as in Horchani et al. (2011). All extraction steps were carried out at $4{ }^{\circ} \mathrm{C}$. Frozen material $(40-60 \mathrm{mg}$ ) was crushed in liquid nitrogen with $300 \mathrm{~mL}$ of perchloric acid solution, containing $7 \%$ (v/v) $\mathrm{HClO}_{4}$ and $25 \mathrm{mM} \mathrm{Na}_{2}$ EDTA, with a mortar and pestle. After thawing, the extract was taken and the mortar was rinsed with $200 \mathrm{~mL}$ of perchloric acid solution, which was then pooled with the extract. The sample was centrifuged for $5 \mathrm{~min}$ at $13,000 \mathrm{~g}$. The supernatant was quickly and carefully neutralized at $\mathrm{pH} 5.6-6.0$ using a $2 \mathrm{M} \mathrm{KOH}-0.3 \mathrm{M}$ MOPS solution. $\mathrm{KClO}_{4}$ precipitate was discarded by centrifugation (5 min, 13,000 g). Adenine nucleotides of the supernatant were measured in a Xenius spectrofluorimeterluminometer (Safas, Monaco) using the ATPlite one-step assay system (ATPLT1STP-0509; Perkin-Elmer) according to the manufacturer's instructions. Proteins were extracted and quantified on clarified extracts as in Horchani et al. (2010).

\section{Results}

\section{Salinity and flooding affect nodulated roots nitrogen-fixing capacity and energy state, and increase NO production}

Salt and flooding treated $M$. sativa roots were first analysed for their biomass and nodule production. As reported in Table S1, root system dry weight is not affected by the two treatments. However, T20 and T50 salt treatments decrease by 38 and $52 \%$, respectively, the number of nodules per plant, indicating that salt stress affects nodulation. On its side, flooding treatment has no effect on the number of nodules per plant. It can be noted that, regardless of the treatment, the protein content of the root system remains unchanged (Fig. S3), suggesting that, as a whole, the root system is not senescent and is still functional.

The effects of salinity and flooding on M. sativa BNF capacity were assessed by measuring the ARA of nodules (Fig. 1a). As compared with control nodules in normoxia, T20 and T50 treatments result in an 18 and 75\% decrease in ARA, respectively. When compared to normoxia, flooding also results in a 33 and 52\% decrease in ARA for control and T20 nodules, respectively, but is without additional effect for T50 nodules.

To test the effects of salinity and flooding on the energy state, we analyzed the ATP/ADP ratio (experiment 1). ATP/ADP ratio of control roots is 7.4 (Fig. 1b), which is in agreement with previous analysis in either roots or nodules (Brouquisse et al. 1991; Horchani et al. 2011). ATP/ADP ratio is not modified in T20 (7.0), but significantly decreased in T50 roots (6.5), indicating that average but not low salt stress impairs the energy status of the nodulated roots. After a 5-day flooding period, ATP/ADP ratios decrease close to 5.0, 4.5 and 4.2 in Ctrl, T20 and T50 treated roots, respectively (Fig. 1b). These results indicate that the saline and flooding stresses, independently of each other, affect the energy state of the nodulated roots and that their combined effect is partly cumulative.

In roots and nodules under hypoxia, NO production has been associated with the establishment of a Phytogb-NO respiration, whose function is to maintain the energy state of the tissues when $\mathrm{O}_{2}$ concentration decreases (Igamberdiev and Hill 2009; Horchani et al. 2011). We checked whether NO production could be involved in the maintenance of the root system energy state under each stress. As compared with control, T20 and T50 treatments caused a 1.8 and 3.8fold increase in NO production (Fig. 1c). Similarly, flooding induced a 1.3 to 2.7 -fold increase in NO production compared to normoxic conditions. As a control, NO production was also measured with $\mathrm{Cu}$ (II) fluorescein probe with similar results (Fig. S3), indicating that measurements with DAF-2 are reliable and relevant in this context. Thus, NO production was increased by both saline and flooding treatments, suggesting (1) that the effects of salinity and flooding on NO production and energy state could result from hypoxic environment, and (2) that Phytogb-NO respiration could be activated in response to both saline and flooding/ hypoxia treatments.

\section{NO production contributes to the maintenance of energy state under both salinity and hypoxia}

To test the above hypothesis, we analyzed the effects of cPTIO (a NO scavenger) and Tg (a NR and Phytogb-NO respiration inhibitor) on ATP/ADP ratio and NO production in the root system of control (0) and T50 (50) treated plants submitted $(\mathrm{H})$ or not $(\mathrm{N})$ to hypoxia. To this end, we 


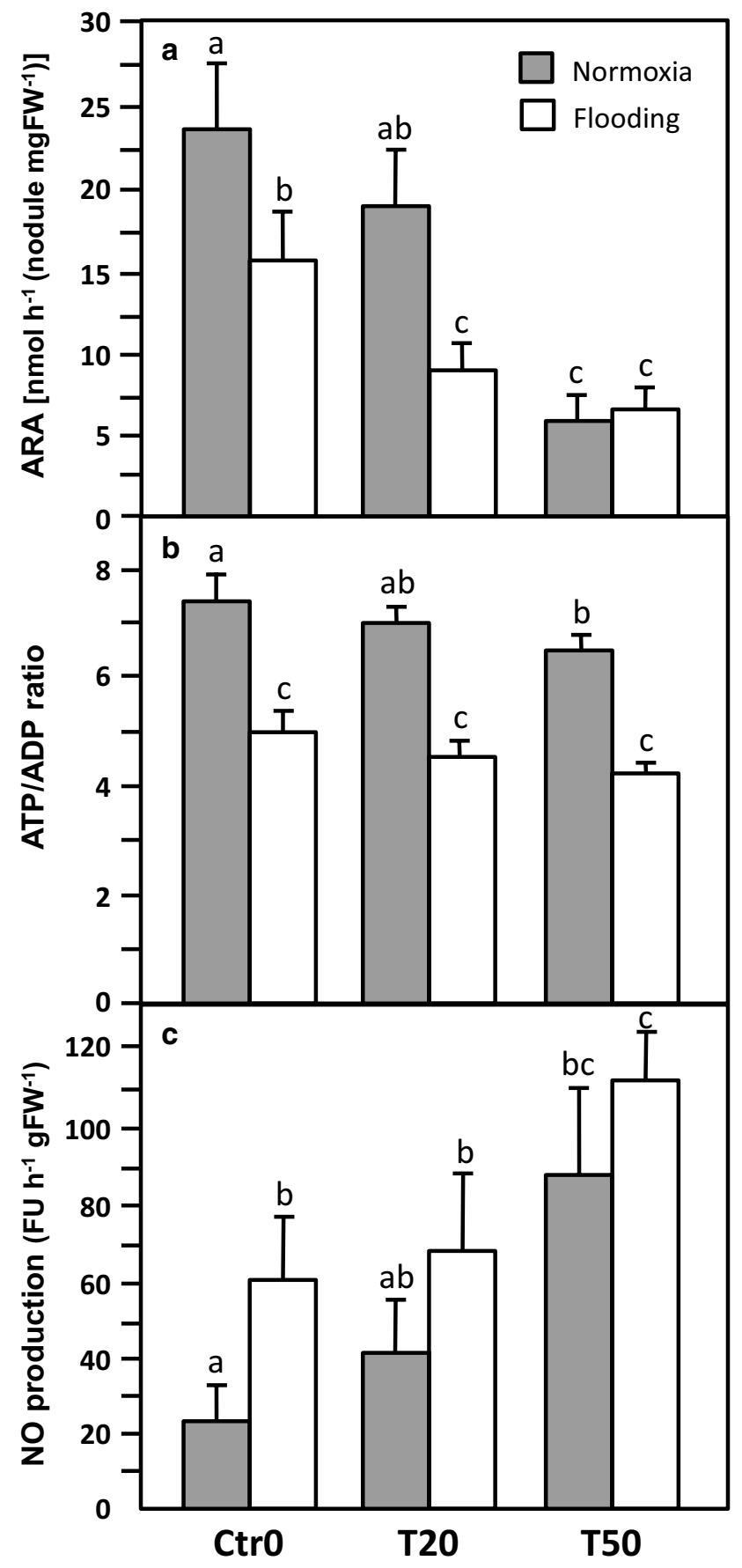

Fig. 1 a Nitrogen-fixing activity, b energy state and $\mathbf{c}$ NO production of Medicago sativa nodulated roots submitted to saline and hypoxic treatments. T20 and T50 refer to 20 and $50 \mathrm{mM} \mathrm{NaCl}$ treatments, respectively. Nitrogen-fixing activity is measured through acethylene reducing activity (ARA). Energy state is evaluated through ATP/ADP ratio. The values are mean $\pm \mathrm{SE}(n=3$ for ATP/ADP ratio; $n=4$ for ARA and NO production) of 4 independent experiments. Values followed by different letters are significantly different according to oneway ANOVA analysis followed by a Tukey test $(P<0.05)$ used a specific hydroponic experimental setup where the root system was incubated in nutrient solution supplemented or not with the inhibitors (experiment 2, Fig. S2). Compared to the control $(0-\mathrm{N})$, NR inhibition by Tg results in a 43,33 and $41 \%$ decrease of ATP/ADP ratio in $0-\mathrm{H}, 50-\mathrm{N}$ and $50-\mathrm{H}$ roots, respectively (Table 1). Similarly, NO scavenging by cPTIO results in a 34,37 and $37 \%$ decrease of ATP/ADP ratio in $0-\mathrm{H}, 50-\mathrm{N}$, and $50-\mathrm{H}$ roots, respectively, compared to control conditions. cPTIO and Tg has no significant effect on the ATP/ADP ratio in $0-\mathrm{N}$ roots. Treatment with Tg triggers a strong decrease in $\mathrm{NO}$ production in $0-\mathrm{H}, 50-\mathrm{N}$ and $50-\mathrm{H}$ treated root systems, but not in $0-\mathrm{N}$ treated root system (Table 1). In the presence of cPTIO, NO production is totally abolished. Taken together, these data indicate that both the functioning of NR and the production of NO are necessary to maintain root and nodule energy state under salinity and hypoxia, alone or combined together.

\section{Discussion}

The present data show that nodulation, but not root growth, of $M$. sativa plants is affected by average salinity (Table $\mathrm{S} 1$ ). This is in line with literature data reporting that alfalfa is a moderately salt-tolerant legume (Bruning and Rozema 2013) and that its growth is significantly affected only from $\mathrm{NaCl}$ concentrations above $80 \mathrm{mM}$ (Rogers et al. 2008, 2009, 2011). However, salinity is known to inhibit BNF more rapidly than growth in legumes (Bruning and Rozema 2013). In our study, ARA is the more inhibited as the salt concentration increases (Fig. 1). Under low salt stress, the supply of $\mathrm{O}_{2}$ to nodules was suggested to be the limiting factor for BNF (Bergersen 1982; Delgado et al. 1994; Serraj et al. 1994). This inhibition would be due to the fact that the salt stress disrupts the symplastic connections between nodule cells, decreases the permeability of the nodules to $\mathrm{O}_{2}$ and increases its critical $\mathrm{O}_{2}$ pressure, which would have the effect of inhibiting the energy metabolism and the regeneration of ATP. These interpretations were supported by Serraj et al. (1998) and Serraj and Drevon (1998) who showed that the BNF of common bean, soybean and alfalfa submitted to $50-100 \mathrm{mM} \mathrm{NaCl}$ concentrations is partly inhibited compared to that of control plants when measured at $21 \% \mathrm{O}_{2}$, but that the inhibition is reversed by increasing $\mathrm{pO}_{2}$. Considered together, these studies suggest that the decrease in ARA under salt stress is due to an $\mathrm{O}_{2}$ limitation within the nodules leading to a reduction in energy metabolism.

Short-term effects of flooding on BNF have been little studied. However, in Trifolium repens (Pugh et al. 1995) and soybean (Sánchez et al. 2010) nodulated roots, a short period of flooding (6-7 days) resulted in a complete or half inhibition of ARA, respectively. Similarly, our data show that in M. sativa nodulated roots, ARA is inhibited after 
5 days of flooding (Fig. 1), confirming that flooding inhibits ARA presumably through flooding-associated $\mathrm{O}_{2}$ shortage.

In the root system of many plant species, including legumes, submitted to flooding or hypoxia, the induction of a Phytogb-NO respiration occurs, allowing cell energy status retention (Dordas et al. 2003; Gupta and Igamberdiev 2011; Berger et al. 2018). The Phytogb-NO respiration cycle involves (1) the reduction of $\mathrm{NO}_{3}{ }^{-}$by NR (2) the translocation of $\mathrm{NO}_{2}^{-}$from the cytosol to the mitochondrial matrix, (3) the reduction of $\mathrm{NO}_{2}^{-}$to $\mathrm{NO}$ via the mitochondrial electron transfer chain (ETC) allowing ATP regeneration, and finally (4) the passive diffusion of NO to the cytosol where it is oxidized back to $\mathrm{NO}_{3}{ }^{-}$by Phytogb (Gupta and Igamberdiev 2011). Functional legume nodules are naturally characterized by a microoxic environment, and the existence of such a Phytogb-NO respiration was also evidenced in $M$. truncatula nodules (Horchani et al. 2011). In these nodules, a hypoxic treatment triggers an increase in NO production, which is abolished by the addition of either cPTIO or Tg, and the energy state (ATP/ADP) is significantly inhibited when nodules are incubated in the presence of $\mathrm{Tg}$ (Horchani et al. 2011). In our study, under either salt or hypoxic treatment, or both, the use of $\mathrm{Tg}$ and cPTIO leads to a reduction of the ATP/ADP ratio and to an inhibition of NO production (Table 1) indicating that both NR activity and NO production are necessary to maintain the energy state. Taken together our data first confirm that in M. sativa nodulated roots under flooding/hypoxia, NO production is involved in maintaining energy state presumably through the establishment of a Phytogb-NO respiration. Second, our study shows that NR-dependent NO production is also involved in maintaining energy state under salt stress, which argues that salt treatment triggers a hypoxic stress in the nodulated roots. Third, this study strongly suggests that the functioning of Phytogb-NO respiration is induced in nodulated roots under salt stress.

Author contributions statement $\mathrm{RB}$ and SA-S planned and designed the research. FA, HS, AG and RB performed experiments and conducted fieldwork. AK, SA-S and RB interpreted the data. RB wrote the manuscript.

Acknowledgements This work was supported by INRAE and by the Comité Mixte Franco-Tunisien pour la Coopération Universitaire (PHC Utique, Grant no. 17G0904).

\section{References}

Bailey-Serres J, Voesenek LACJ (2008) Flooding stress: acclimations and genetic diversity. Annu Rev Plant Biol 59:313-339. https:// doi.org/10.1146/annurev.arplant.59.032607.092752 
Berger A, Brouquisse R, Pathak PK, Hichri I, Singh I et al (2018) Pathways of nitric oxide metabolism and operation of phytoglobins in legume nodules: missing links and future directions. Plant Cell Env 41:2057-2068. https://doi.org/10.1111/pce.13151

Bergersen FJ (1982) Root nodules of legumes: Structure and functions. Research Studies Press/Wiley, Chichester

Besson-Bard A, Pugin A, Wendehenne D (2008) New insights into nitric oxide signaling in plants. Annu Rev Plant Biol 59:21-39. https://doi.org/10.1146/annurev.arplant.59.032607.092830

Blokhina O, Fagerstedt KV (2010) Oxidative metabolism, ROS and NO under oxygen deprivation. Plant Physiol Biochem 48:359-373. https://doi.org/10.1016/j.plaphy.2010.01.007

Brouquisse R, James F, Raymond P, Pradet A (1991) Study of glucose starvation in excised maize root tips. Plant Physiol 96:619-626. https://doi.org/10.1104/pp.96.2.619

Bruning B, Rozema J (2013) Symbiotic nitrogen fixation in legumes: perspectives for saline agriculture. Env Exp Bot 92:134-143. https ://doi.org/10.1016/j.envexpbot.2012.09.001

del Giudice J, Cam Y, Damiani I, Fung-Chat F, Meilhoc E, Bruand C, Brouquisse R, Puppo A, Boscari A (2011) Nitric oxide is required for an optimal establishment of the Medicago truncatulaSinorhizobium meliloti symbiosis. New Phytol 191:405-417. https ://doi.org/10.1111/j.1469-8137.2011.03693.x

Delgado MJ, Ligero F, Lluch C (1994) Effects of salt stress on growth and nitrogen fixation by pea, faba-bean, common bean and soybean plants. Soil Biol Biochem 26:371-376. https://doi. org/10.1016/0038-0717(94)90286-0

Dordas C, Hasinoff BB, Igamberdiev AU, Manac'h N, Rivoal J, Hill RD (2003) Expression of a stress-induced hemoglobin affects NO levels produced by alfalfa root cultures under hypoxic stress. Plant J 35:763-770. https://doi.org/10.1046/j.1365-313x.2003.01846.x

Gupta KJ, Igamberdiev AU (2011) The anoxic plant mitochondrion as a nitrite: NO reductase. Mitochondrion 11:537-543. https://doi. org/10.1016/j.mito.2011.03.005

Hartman S, Liu Z, van Veen H, Vicente J, Reinen E et al (2019) Ethylene-mediated nitric oxide depletion pre-adapts plants to hypoxia stress. Nat Commun 10:4020. https://doi.org/10.1038/s4146 7-019-12045-4

Hichri I, Boscari A, Castella C, Rovere M, Puppo A, Brouquisse R (2015) Nitric oxide: a multifaceted regulator of the nitrogen-fixing symbiosis. J Exp Bot 66:2877-2887. https://doi.org/10.1093/jxb/ erv051

Horchani F, Hajri R, Khayati H, Brouquisse R, Aschi-Smiti S (2010) Does the source of nitrogen affect the response of subterranean clover to prolonged root hypoxia? J Plant Nutr Soil Sci 173:275283. https://doi.org/10.1002/jpln.200900280

Horchani F, Prevot M, Boscari A, Evangelisti E, Meilhoc E et al (2011) Both plant and bacterial nitrate reductases contribute to nitric oxide production in Medicago truncatula nitrogen-fixing nodules. Plant Physiol 155:1023-1036. https://doi.org/10.1104/ pp. 110.166140

Igamberdiev AU, Hill RD (2009) Plant mitochondrial function during anaerobiosis. Ann Bot 103:259-268. https://doi.org/10.1093/aob/ $\operatorname{men} 100$

Kopyra M, Gwózdz EA (2003) Nitric oxide stimulates seed germination and counteracts the inhibitory effect of heavy metals and salinity on root growth of Lupinus luteus. Plant Physiol Biochem 41:1011-1017. https://doi.org/10.1016/j.plaphy.2003.09.003

Lim MH, Xu D, Lippard SJ (2006) Visualization of nitric oxide in living cells by a copper-based fluorescent probe. Nat Chem Biol 2:375-380. https://doi.org/10.1038/nchembio794

Liu Y, Wu R, Wan Q, Xie G, Bi Y (2007) Glucose-6-phosphate dehydrogenase plays a pivotal role in nitric oxide-involved defense against oxidative stress under salt stress in red kidney bean roots. Plant Cell Physiol 48:511-522. https://doi.org/10.1093/pcp/ pcm020
Molassiotis A, Tanou G, Diamantidis G (2010) NO says more than 'YES' to salt tolerance salt priming and systemic nitric oxide signaling in plants. Plant Signal Behav 5:209-212. https://doi. org/10.4161/psb.5.3.10738

Pierre O, Hopkins J, Combier M, Baldacci F, Engler G et al (2014) Involvement of papain and legumain proteinase in the senescence process of Medicago truncatula nodules. New Phytol 202:849-863. https://doi.org/10.1111/nph.12717

Pugh R, Witty JF, Mytton LR, Minchin FR (1995) The effect of waterlogging on nitrogen fixation and nodule morphology in soil-grown white clover (Trifolium repens L.). J Exp Bot 46:285-290. https://doi.org/10.1093/jxb/46.3.285

Rogers ME, Colmer TD, Frost K, Henry D, Cornwall D et al (2008) Diversity in the genus Melilotus for tolerance to salinity and waterlogging. Plant Soil 304:89-101. https://doi.org/10.1007/ s11104-007-9523-y

Rogers ME, Colmer TD, Frost K, Henry D, Cornwall D et al (2009) The influence of $\mathrm{NaCl}$ salinity and hypoxia on aspects of growth in Trifolium species. Crop Past Sci 60:71-82. https:// doi.org/10.1071/CP08123

Rogers ME, Colmer TD, Nichols PGH, Hughes SJ, Frost K et al (2011) Salinity and waterlogging tolerance amongst accessions of messina (Melilotus siculus). Crop Past Sci 62:225-235. https ://doi.org/10.1071/CP10270

Sánchez C, Gates AJ, Meakin GE, Uchiumi T, Girard L, Richardson DJ, Bedmar EJ, Delgado MJ (2010) Production of nitric oxide and nitrosylleghemoglobin complexes in soybean nodules in response to flooding. Mol Plant-Mi Inter 23:702-711. https:// doi.org/10.1094/MPMI-23-5-0702

Serraj R, Drevon JJ (1998) Effects of salinity and nitrogen source on growth and nitrogen fixation in alfalfa. J Plant Nutr 21:18051818. https://doi.org/10.1080/01904169809365525

Serraj R, Roy G, Drevon JJ (1994) Salt stress induces a decrease in the oxygen uptake of soybean nodules and their permeability to oxygen diffusion. Physiol Plant 91:161-168. https://doi. org/10.1111/j.1399-3054.1994.tb00414.x

Serraj R, Vasquez-Diaz H, Drevon JJ (1998) Effects of salt stress on nitrogen, fixation, oxygen diffusion, and ion distribution in soybean, common bean and alfalfa. J Plant Nutr 21:475-488. https://doi.org/10.1080/01904169809365418

Sghaier H, Aloui A, Khadri A, Aschi-Smiti S, Brouquisse R (2020) Effects of combined salt and flooding stresses on the growth and physiological behavior of alfalfa (Medicago sativa L.). Afric J Agro Res 16:758-764. https://doi.org/10.5897/AJAR2 020.14831

Simontacchi M, Galatro A, Ramos-Artuso F, Santa-María G (2015) Plant survival in a changing environment: the role of ritric oxide in plant responses to abiotic stress. Front Plant Sci 6:977. https:// doi.org/10.3389/fpls.2015.00977

Stoimenova M, Igamberdiev AU, Gupta KJ, Hill RD (2007) Nitritedriven anaerobic ATP synthesis in barley and rice root mitochondria. Planta 226:465-474. https://doi.org/10.1007/s0042 5-007-0496-0

Tanou G, Molassiotis A, Diamantidis G (2009) Hydrogen peroxideand nitric oxide-induced systemic antioxidant prime-like activity under $\mathrm{NaCl}$-stress and stress free conditions in citrus plants. J Plant Physiol 166:1904-1913. https://doi.org/10.1016/j.jplph .2009 .06 .012

Thalineau E, Truong H-N, Berger A, Fournier C, Boscari A et al (2016) Cross-regulation between $\mathrm{N}$ metabolism and nitric oxide (NO) signaling during plant immunity. Front Plant Sci 7:472. https:// doi.org/10.3389/fpls.2016.00472

Uchida A, Jagendorf AT, Hibino T, Takabe T, Takabe T (2002) Effects of hydrogen peroxide and nitric oxide on both salt and heat stress tolerance in rice. Plant Sci 63:515-523. https://doi.org/10.1016/ S0168-9452(02)00159-0 
Wang H, Liang X, Wan Q, Wang X, Bi Y (2009) Ethylene and nitric oxide are involved in maintaining ion homeostasis in Arabidopsis callus under salt stress. Planta 230:293-307. https://doi. org/10.1007/s00425-009-0946-y

Wany A, Kumari A, Gupta KJ (2017) Nitric oxide is essential for the development of aerenchyma in wheat roots under hypoxic stress. Plant Cell Environ 40:3002-3017. https://doi.org/10.1111/ pce. 13061

Zhang Y, Wang L, Liu Y, Zhang Q, Wei Q, Zhang W (2006) Nitric oxide enhances salt tolerance in maize seedlings through increasing activities of proton-pump and $\mathrm{Na}^{+} / \mathrm{H}^{+}$antiport in the tonoplast. Planta 224:545-555. https://doi.org/10.1007/s00425-006-0242-Z
Zhao G, Zhao Y, Yu X, Kiprotich F, Han H, Guan R, Wang R, Shen W (2018) Nitric oxide is required for melatonin-enhanced tolerance against salinity stress in rapeseed (Brassica napus $L$ ) seedlings. Int J Mol Sci 19:1912. https://doi.org/10.3390/ijms19071912 